\title{
Innovative Manufacturing and Application of Fiber Metal Laminate Pipe
}

\author{
Zijian Lang ${ }^{1,}$, Meng Zhang ${ }^{2}$, Xiaoxing Li $^{2}$ and Xing Huang ${ }^{1}$ \\ ${ }^{1}$ Beijing University of Civil Engineering and Architecture,No. 15 Yongyuan Road, DaXing District, Beijing, P.R. China \\ ${ }^{2}$ Beihang University, No. 37 Xueyuan Road, Haidian District, Beijing, P.R. China
}

\begin{abstract}
Fiber-reinforced metal laminate tube (FMLT) is a multi-layered ultra-hybrid material that cures at fixed pressure and temperature after alternating metal laminates and fiber composites. As with the production process similar to the second-generation GLARE fiber-reinforced metal layer, the management layer of the formed composite layer should have good impact resistance characteristics, and can also be used in the impact-resistant structure of the aircraft, the landing cushion structure of the aircraft, and the body collision Device to protect aviation materials. The composite pipe fittings are made by hydraulic forming technology, which lays a good foundation for the small-scale fine processing of the pipe. In addition, use speckle models and other virtual software simulation models (such as various related software and related formulas) to monitor data before and after hydroforming of fiber-reinforced metal layer tubes, it lays a good data foundation for the hydroforming of pipes and subsequent experiments. The development and performance testing of GLARE composite tube hydraulic forming technology is of great significance to the development of lightweight and safety in the aviation industry and the automobile industry.
\end{abstract}

\section{Introduction}

Due to its small density and corrosion resistance, aluminum alloy has always been the first choice to reduce the quality of the structure, especially in the aerospace industry, automotive and high-speed rail, and other transportation industry manufacturing industries. With the rapid development of the domestic aviation and aerospace industries and the rapid development of household automobiles, aluminum alloy pipe fittings have become more and more widely used in the above industries [1]. The successful application of Tube Hydroforming-THF technology in the automotive industry will greatly promote the rapid development of the technology in aerospace, weapon manufacturing, and civilian and other related industries [2]. Especially for airplanes, rockets, missiles and engines, and other related manufacturing industries, the hollow thin-walled tube forming technology with complex cross-sections of new composite materials requires a compact layout and extremely urgent lightweight requirements. In addition, in order to further reduce the weight of design, production, and target mix ratio, ratio and impact resistance, we have proposed the hydraulic expansion of hydraulically expanded metal fiber composite layer pipes to replace key absorbent parts and related traditional metal pipes. Lightweight components.

\subsection{Research background}

With similar production of second-generation GLARE fiber-reinforced metal layer production process, the formation of composite layer management should have good anti-impact performance characteristics, but also can be used for aircraft impact-resistant structure, aircraft landing buffer structure, car body anti-collision device, air-borne material protection.

At the same time, due to its excellent corrosion resistance, it can be widely used in the chemical industry. The development of GLARE composite laminate technology and performance testing is of great significance for the development of lightweight and safety in the aviation industry and the automotive industry.

This paper through several aspects of theoretical research, so that the production of fiber metal layer tube and follow-up research has a general research and production ideas. The structure is shown in the following image Fig. 1:

\section{Research objectives and content}

\footnotetext{
* Corresponding author: lzileo@163.com
} 


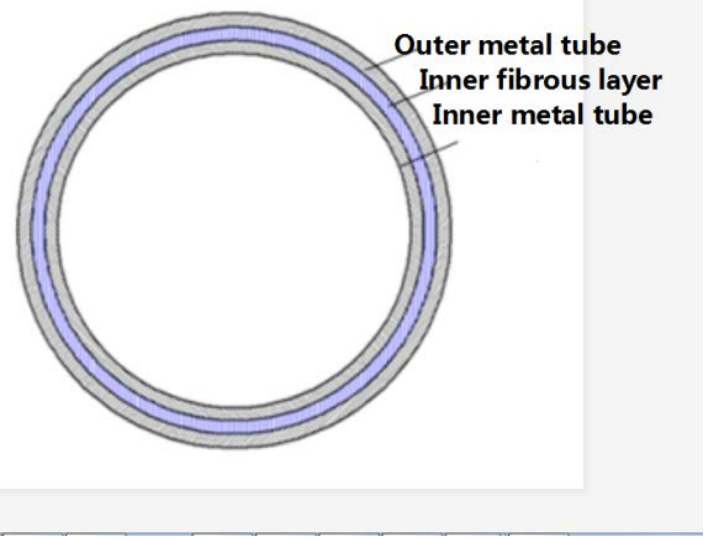

Fig. 1. Structural sketch of GLARE layered tube

\subsection{Research objectives}

Through basic test and theoretical analysis, the optimal design of the expansion key mold, sealing device and key process parameters is completed, and the corresponding preparation general process path is established. Based on the mechanical performance test of the existing pipe, it is suggested that a pipe bloating test method based on the linear loading of the strain ratio is proposed. Secondary development using abaqus software, the actual strain (LE)at the highest point in the tube FLD test is within the set interval to solve the problem of the strain ratio changing with the load in the existing pipe FLD test.

In addition, the use of physical speckle experiments and one-way stretching experiments, and the establishment of related theories, through detailed theoretical basis for the subsequent accurate numerical simulation to provide solutions.

\subsection{Research content}

\subsubsection{The hydraulic preparation process and related mold design for fiber metal laminate.}

First of all, the new laminated materials need to be prepared by manual method, the process includes aluminum alloy surface treatment, manual placement of pre-immersion material (glass fiber continuous braiding) and vacuum treatment, unlike the traditional fiber metalreinforced sheet, fiber metal-reinforced tube preparation needs to be deformed to ensure close contact between the two layers of metal tube. In order to ensure that the inner tube changes uniform, the internal and external tubes are tightly combined, this topic adopts the hydraulic bloating way. In order to ensure that the outer tube is always inelastic deformation in the process of expansion, it is necessary to complete the key expansion mold and related sealing device and to complete the establishment and optimization of the key parameters of multi-layer tube expansion through theoretical calculation and numerical simulation technology. The simulated diagram is shown in the following image Fig. 2 and Fig. 3:

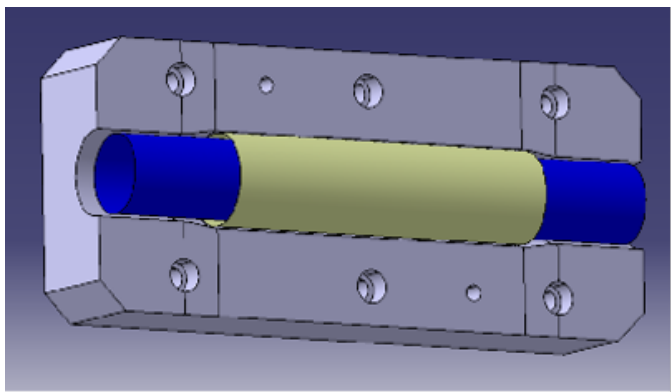

Fig. 2. Mold design of fiber metal layer tube

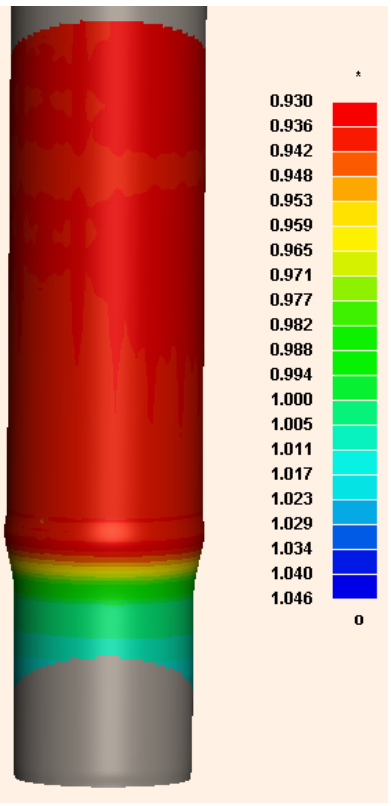

Fig. 3. Mold-based data simulation of fiber metal layer tubes

\subsubsection{Path optimization for thin-walled pipe FLD test}

Through numerical simulation technology, the path optimization of the tube FLD performance test in different strain ratio states is established [3]. By controlling the matching relationship between the axial feed and internal pressure of the two ends of the pipe in the TUBE FLD test, the ratio of axial strain to the peripheral strain is within the target's certain threshold, and the 5A02 aluminum alloy tube FLD test is completed, which provides the tube mechanical performance parameters for the numerical simulation of the pipe expansion in the upper sequence.

\subsubsection{Speckle experiment and one-way stretching test}

The speckle experiment can directly observe the expansion degree of the pipe by visual effect, and the one-way stretch test can reveal the characteristics and basic laws of the stress-strain relationship and the common three failure forms (excessive elastic 
deformation, plastic deformation, and fracture) of the material under static load.

\subsection{Hydraulic bloating test and FLD test of pipe}

Compared with the traditional tube performance test, the pipe bloating test can obtain the structural equation and forming limit of the pipe under the action of the fluid surface force more accurately. In this paper [4], taking 5A02 aluminum alloy tube as an example, using the three-dimensional digital spot-related method(3D-DIC) to obtain the bloated profile, get the pipe structure equation and the internal contour curve, the pipe expansion test system as shown in Fig. 4 below:

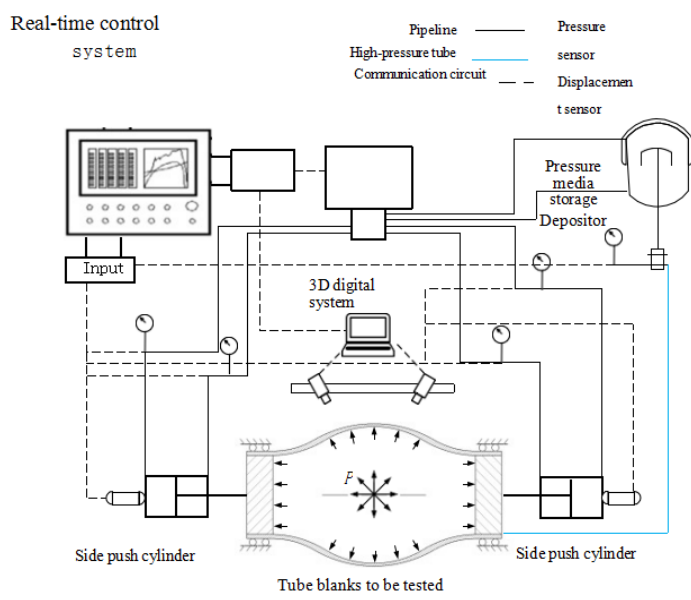

Fig. 4. Bulging test system

The flat profile is measured by the three-dimensional digital spot correlation method and fitted with different contour curves as shown in Fig. 5 below:

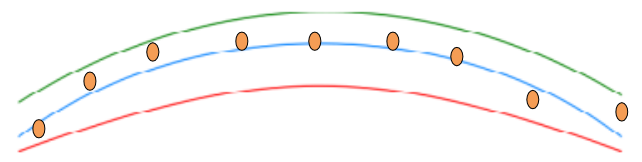

Discrete points $0 \quad \operatorname{Tim} \longrightarrow$ Cosin $\longleftarrow$ Ellipti

Fig. 5. Contour curve fit

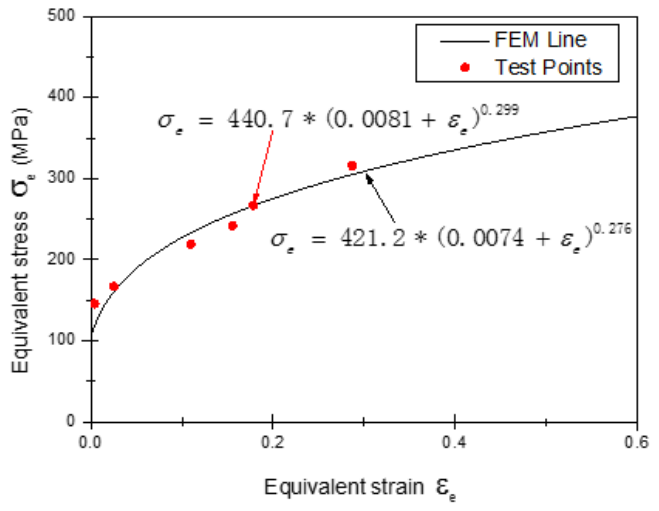

Fig. 6. 5A02 Aluminum Structure Equation
It can be seen that fitting the 5A02 aluminum alloy swell profile curve with a cosine curve is better, and on this basis, the final structure equation of 5A02 aluminum alloy is obtained through the calculation of axial and peripheral strain, as shown in Fig. 6.

For tube bloating tests, the mechanical model can be represented by Fig. 7 .

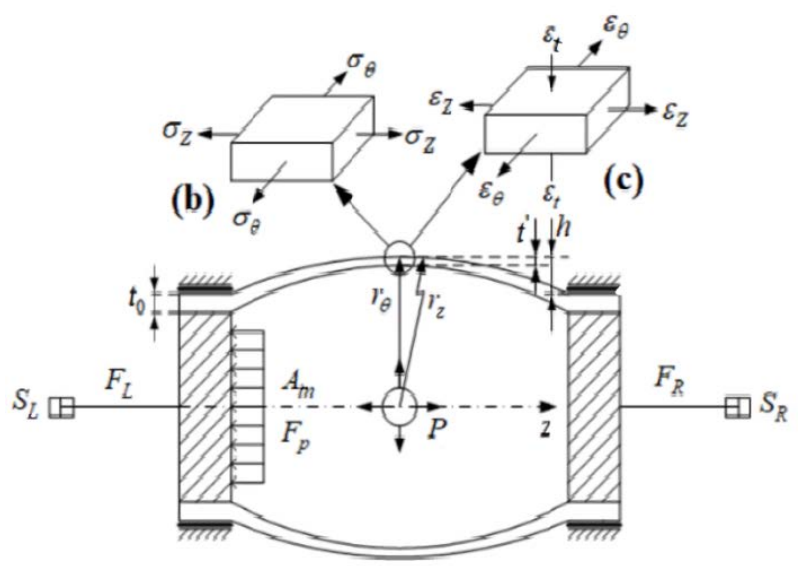

Fig. 7. Mechanical model of pipe bloating test

\section{Tube blank-related experiments and their theoretical research}

\subsection{Speckle experiment}

The intercept length is $550 / 300 \mathrm{~mm}$ (inside/outside) as a preparation for a free-expansion experiment. Rounded treatment is performed on both ends of the tube, and the outside of the pipe is sprayed with spotting, as shown in the following Fig. 8.

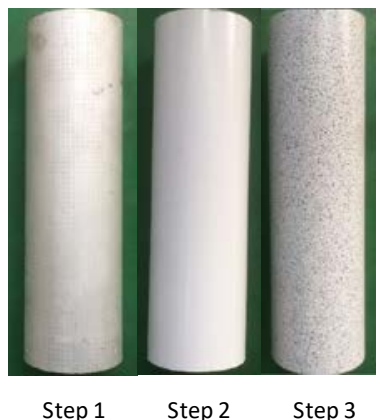

Fig. 8. Flat experimental tube blank preparation

The pipe is fixed and sealed, first of all, the spot calibration, as shown in the following figure. After that, the pressure is carried out, the tube with the internal pressure of the continuous larger and plastic deformation until the pipe rupture [5].

\subsection{One-way stretch test}

The forming performance index obtained by the one-way stretch experiment is called the basic forming performance index, through which the material's elastic modulus E, yield strength $\mathrm{Rp}$, tensile strength $\mathrm{Rm}$, 
uniform elongation A, limit elongation A, anisotropic index $r$ value, strain hardening index $n$ value can be obtained In numerical simulation, the strain hardening coefficient $\mathrm{K}$ value, strain rate sensitivity index $\mathrm{m}$ value, $\mathrm{Qu}$-force ratio $\mathrm{Rp} / \mathrm{Rm}$, broken cross-section shrinkage Z, etc., in numerical simulation, the construction of the material structure model also depends on these parameters, especially $\mathrm{Rp}$, r value, $n$ Value, $\mathrm{K}$ value. The specimen specification of the single pull test to take the ordinary one-way stretch test is shown in the following image, and the single-pull test is made to carry out the single-pull test. Fig. 9 shows the single-pull test.

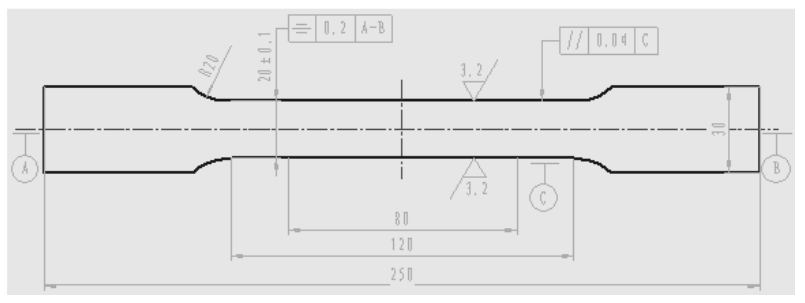

Fig. 9. Single-pull test

In numerical simulation, low simulation accuracy is the main aspect that limits its application, and the material structure relationship is one of the main factors affecting the accuracy of numerical simulation [6]. In this structure relationship, if the initial yield face of the material is determined, that is, the yield criterion is determined, then combined with a certain strengthening law, the corresponding structure relationship can be derived, and after the structure relationship is determined, the stress and strain behavior of the material in the deformation process can also be predicted.

1) Establishment of yield criterion

The pipe slab used in the filling of the pipe is welded by the plate, which undergoes many roll and heat treatment during production, generally has a certain deformation orientation and presents a clear anisotropy, which has a significant impact on the plastic denature behavior. The main stress of the tube in hydraulic bloating - the strain state is the plane stress state, Barlat and Lian proposed in 1989 to consider the anisotropy in the plane under flat stress conditions, the yield guideline [7], which accurately describes the use of Bishop and Hill The yield trajectory of the crystal material model, as shown in the equation(1):

$$
\begin{aligned}
& f=a\left|K_{1+} K_{2}\right|^{m}+a\left|K_{1}-K_{2}\right|^{m}+(2-a)\left|2 K_{2}\right|^{m}-2 \sigma_{s}^{m} \\
& K_{1}=\left(\sigma_{x x}+h \sigma_{y y}\right) / 2 K_{2}=\sqrt{\left(\left(\sigma_{x x}-h \sigma_{y y}\right) / 2\right)^{2}+p^{2} \sigma_{x y}^{2}}
\end{aligned}
$$

In this formula, $\mathrm{m}$ is a non-secondary yield function index; $\mathrm{x}, \mathrm{y}$, and $\mathrm{z}$ are parallel to the rolling direction and perpendicular to the rolling direction, perpendicular to the plate plane direction; $a, h, p$ is the material parameter that characterizes anisotropy, there are two calculation methods, one is calculated according to stress calculation, i.e. using the method of stress calculation Bishop and Hill material models calculate stresses under different loading conditions to obtain these parameters. For example, assume $90 \mathrm{~F} 1$ is yield stress when it is pulled in a single pull direction of 90 degrees with the rolling direction, if $\tau \mathrm{s} 1, \tau \mathrm{s} 2$ is a pure shear yield stress, when $\sigma_{\mathrm{yy}}=-\sigma_{\mathrm{xx}}=\tau_{\mathrm{s} 2}$, then $\sigma_{\mathrm{xy}}=0$, when $\sigma_{\mathrm{xx}}=\sigma_{\mathrm{yy}}=0$, then $\sigma_{\mathrm{xy}}=\tau_{\mathrm{s} 1}$, meantime:

$$
\begin{gathered}
h=\frac{\bar{\sigma}}{\sigma_{90}} \\
p=\frac{\bar{\sigma}}{\tau_{s 1}}\left(\frac{2}{2 a+2^{m}(2-a)}\right)^{1 / m} \\
\mathrm{a}=\left(2\left(\frac{\bar{\sigma}}{\tau_{s 2}}\right)^{m}-2\left(1+\frac{\bar{\sigma}}{\sigma_{90}}\right)^{m}\right)\left(1+\left(\frac{\bar{\sigma}}{\sigma_{90}}\right)^{m}-\left(1+\frac{\bar{\sigma}}{\sigma_{90}}\right)^{m}\right)
\end{gathered}
$$

Another method is calculated based on the thick anisotropy index r0, r45, r90:

$$
a=2-2 \sqrt{\frac{r_{0}}{1+r_{0}} \cdot \frac{r_{90}}{1+r_{90}}} h=\sqrt{\frac{r_{0}}{1+r_{0}} \cdot \frac{1+r_{90}}{r_{90}}}
$$

At first the p-value cannot be resolved, But when the values of a, c and h are known, it is verified that for the single-axis stretch, $\mathrm{r} \Phi(\mathrm{A}$ thick anisotropy index in three directions: $0^{\circ}, 45^{\circ}$ and $\left.90^{\circ}\right) \mathrm{p}$ are single value relations, So it can be calculated by the formula (7) in an iterative manner.

$$
\frac{2 m \sigma_{s}^{m}}{\left(\frac{\partial f}{\partial \sigma_{x x}}+\frac{\partial f}{\partial \sigma_{y y}}\right) \sigma_{45}}-1-r_{45}=g(p)
$$

In this formula, $\sigma 45$ represents yield strength at a single pull with the rolling direction of $45^{\circ}$; For the facecentered cube material, $\mathrm{m}=8$,For body-centered cube materials, $\mathrm{m}=6$.

Verified by Lege et al. The material model using this yield criterion proposed by Barlat-Lian can better predict the strength change steam and forming limits of the 2008-T4 plate, especially when describing $r$ changes.

2)Hardening model establishment

The stress-strain relationship of the material can be expressed in the form of a relationship between the strain reinforcement index $\mathrm{n}$ and the coefficient $\mathrm{k}$ :

$$
\sigma_{\mathrm{y}}=k \varepsilon^{n}=k\left(\varepsilon_{y p}+\bar{\varepsilon}^{p}\right)^{n}
$$

In this formula, $y, p$ refers to the elastic strain of the material when it yields, and is an effective plastic strain. If the initial yield strain SIGY is zero, the strain in the event of yield can be derived from the line elastic stress strain equation and the strain hardening equation: $\sigma=E \varepsilon$.

Strain in the event of submission of the material is: 


$$
\varepsilon_{\text {ур }}=\left(\frac{E}{k}\right)^{\left[\frac{1}{n-1}\right]}
$$

If the initial yield stress SIGY is not equal to zero, and greater than 0.02 , then

$$
\varepsilon_{\text {yp }}=\left(\frac{\sigma_{\text {y }}}{k}\right)^{\left[\frac{1}{n}\right]}
$$

Expansion calculation, $\mathrm{R}$ for the outer tube, $\mathrm{r}$ for the inner tube, the lower corner label $\mathrm{i}$, o represents the inner and outer wall, represents the circumference, $r$ represents axial; According to Lame equation:

$$
\left\{\begin{array}{l}
\sigma_{r}=\frac{p_{i} r_{i}^{2}}{r_{o}^{2}-r_{i}^{2}}\left(1-\frac{r_{o}^{2}}{r_{i}^{2}}\right) \\
\sigma_{\theta}=\frac{p_{i} r_{i}^{2}}{r_{o}^{2}-r_{i}^{2}}\left(1+\frac{r_{o}^{2}}{r_{i}^{2}}\right) \\
u_{r}=\frac{p_{i} r_{i}^{2}}{E_{i}\left(r_{o}^{2}-r_{i}^{2}\right)}\left[\left(1+u_{i}\right) \frac{r_{o}^{2}}{r}+\left(1-u_{i}\right) r\right]
\end{array}\right.
$$

The difference between the first main stress and the third main stress at the inner wall in the upper formula is the largest, and taking into account the Von Mises yield criteria, the limit pressure of the inner tube can be obtained:

$$
p_{e i}=\frac{\sigma_{s i}}{\sqrt{3}}\left(1-\frac{r_{i}^{2}}{r_{o}^{2}}\right)
$$

Consider the inner tube into the plastic and elastic coexistence state, take into the deformation equation, integral:

$$
\sigma_{\theta}-\sigma_{r}=r \frac{d \sigma_{r}}{d r}
$$

Boundary conditions at the inner wall of the inner tube, when $\mathrm{r}=\boldsymbol{r}_{\boldsymbol{i}}$ then $\sigma_{r}=-\mathrm{p}$; and bring the yield criterion into the following formula:

$$
\left\{\begin{array}{l}
\sigma_{r}=\frac{2}{\sqrt{3}} \sigma_{s i} \ln r-p_{i} \\
\sigma_{\theta}=\frac{2}{\sqrt{3}} \sigma_{s i}\left(1+\ln \frac{r}{r_{i}}\right)-p_{i}
\end{array}\right.
$$

The same consideration of the external tube, and the use of internal and external tube deformation coordination conditions, you can get the pressure of the outer tube to reach the elastic limit:

$$
p_{e 0}=\frac{\sigma_{s 0}}{\sqrt{3}}\left(1-\frac{R_{i}^{2}}{R_{0}^{2}}\right)
$$

When considering unloading the same amount of response to the inner and outer tubes, that is $\Delta u_{r 0}=\Delta u_{R i}$, residual contact pressure can be obtained:

$$
p_{d}^{\prime}=(1-2 C) p_{i}-\frac{2}{\sqrt{3}} \sigma_{s i} \ln \frac{r}{r_{i}}
$$

In this formula $\mathrm{c}$ is the constant determined by the internal and external tube material and collection parameters, calculated as:

$$
C=\left\{1+u_{i}+\frac{r_{o}^{2}}{r_{i}^{2}}\left(1-u_{i}\right)+\frac{E_{i}\left(\frac{r_{o}^{2}}{r_{i}^{2}}-1\right)}{E_{o}\left(\frac{R_{o}^{2}}{R_{i}^{2}}-1\right)}\left[1-u_{o}+\frac{R_{o}^{2}}{R_{i}^{2}}\left(1+u_{i}\right)\right]\right\}
$$

\section{Conclusions}

A new type of metal tube fiber has been successfully studied and formed. The results show that the pipeline has higher strength, specific modulus and corrosion resistance than conventional composite metal materials.

As a plastic molding method, the convex shape of the composite pipe can greatly improve the utilization rate and accuracy of the molding material. In addition, the pipe joint has the advantages of a uniform expansion force, uniform wall thickness, and high surface quality. The parameters and mechanical structure of the two metal pipes can be calculated.

To succeed, the necessary theoretical research must be carried out. The above experiments are related to experimental techniques and methods, and provide theoretical research methods. Further research on this experiment is still ongoing.

\section{References}

1. Jia meihui, Tang chengtong. Study on springback prediction model for bending forming of stainless steel pipe [J]. Journal of Beijing Institute of Technology. 2012,32(9):910-914

2. Endow J, Murofa T. flattening of the circular tube section at uniform bending [A]. World Plastic Processing Recent Technology Translation Set

3. Wang ping. Design Reference Aircraft Boeing 7878 Dual-Flower Remote Aircraft [J]. Civil Aircraft Design and Research,2010,(2):37-37.

4. Liu cheng. GLARE Interlaminar shear failure behavior and mechanism study [D]. Nanjing University of Aeronautics and Astronautics,2017.

5. Zhang meng, Lang lihui, Li xiaoxing. tfld test of tube expansion and optimized path based on digital speckle method,2019. journal of aviation.

6. Yan huigeng, Shi tingrui. Liquid bag hydraulic expansion technology of heat exchanger and its application [J]. Chemical Machinery,1998,25(6):339341.

7. Bciht F, LUn J. Plastic behaviour and stretchability of sheet meuls, Part I A yield function for orthotropic 
iheet under plane stress conditions [J] . International Journal of Hutidty. 1969. 5 (l):51-6S. 